\title{
A New Approach for Representing Photonic Crystal Fiber Index Profile to Determine their Optical Characteristics
}

\author{
Maan M. Shaker, Mahmood Sh. Majeed and Raid W. Daoud \\ Techniques of Computer Engineering \\ Technical College \\ Mosul, Iraq \\ Raid.daoud@asiacell.com
}

\begin{abstract}
A genetic algorithm is implemented to represent the different shapes of Photonic Crystal Fiber (PCF) profile; this is due to the fact that such fibers have special structure. A novel approach is suggested which differs from the usual fibers. By applying this new approach, more realistic representations of their profiles are drawn and hence highly accurate results are obtained including propagation and dispersion characteristics.
\end{abstract}

\section{INTRODUCTION}

Photonic Crystal Fibers (PCF), which are optical fibers with a cross-sectional microstructure of air holes and/or doped sections, have in recent years attracted much scientific and technological interest. PCFs offer a number of novel design options, such as very large or very small mode areas, high numerical aperture, guidance of light in air, and novel dispersion properties [1].

Based on pure silica glass, the invention of single material photonic crystal fiber also known as holy fibers (HFs) or microstructure fibers has attracted wide interest over the past few years. The air-filled holey cladding leads to unique guidance properties such as photonic band-gaps at optical wavelengths, very-large-core with endless single mode guidance and soliton effects, polarization maintenance and high birefringence and dispersion management arise and promise various novel applications [2].

\section{THEORY}

The core of the PCF is made of solid silica and the cladding consists of multiple layers of air holes separated by narrow silica bridges. Fig. 1 illustrates an example of the cross-section of an index-guided photonic crystal fiber [3]. The idealized structure of the PCF have regular hexagonal array of perfectly circular air holes.

In recent years, photonic crystal fiber made of pure silica and air holes provided a new approach of dispersion compensation: large negative dispersion (D) can be achieved in PCF due to high relative difference $(\Delta)$ between air and silica, and can be tailored by the size and structure of air holes in the cladding [4]. To determine the dispersion parameters in the conventional fiber the most important thing that must be fixed is the relation between the normalized propagation constant (B), and the $\mathrm{V}$-number. The formula of the normalized propagation constant is given by [4]:

$$
B=\frac{(\beta / k)^{2}-n_{c l}^{2}}{n_{c o}^{2}-n_{c l}^{2}}
$$

where $\beta$ is the propagation constant and must be within the range $\left(\mathrm{kn}_{\mathrm{cl}} \leq \beta \leq \mathrm{kn}_{\mathrm{co}}\right), \mathrm{k}$ is the wave number $(\mathrm{k}=2 \pi / \lambda)$, $\lambda$ is the wavelength while $\mathrm{n}_{\mathrm{co}}$ and $\mathrm{n}_{\mathrm{cl}}$ are the core and the cladding index, respectively.

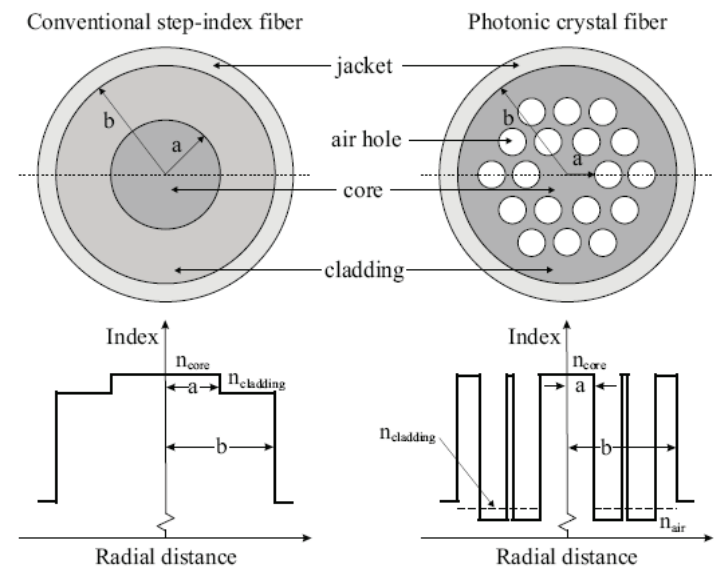

Fig. 1. Schematic illustration of the cross-section and the refractive index profile for an index-guiding photonic crystal fiber. 
Due to the high similarity between the $n_{\mathrm{cl}}$ and $n_{\text {eff }}$ most researchers assume that they are the same. On the other hand, formulation of the V-parameter suggested to be based on an approximation with an equivalent step index (SIF). In the case of a SIF, the V-parameter, VSIF, as function of wavelength, $\lambda$, is given by [3]:

$$
V_{S I F}(\lambda)=\frac{2 \pi}{\lambda} a \sqrt{n_{c o}^{2}-n_{c l}^{2}}
$$

The refractive indices of the core and cladding, $\mathrm{n}_{\mathrm{co}}$ and $\mathrm{n}_{\mathrm{cl}}$, respectively, are assumed to be wavelength independent and ' $a$ ' denotes the core radius. One problem with using the step index approximation for describing the PCF is that the core region is not circular, complicating the definition of values for the refractive indices, is the problematic due to the strong wavelength dependency of the effective indices caused by the air-glass matrix. For these reasons, the V-parameter of the PCF, VPCF is instead proposed to be given as [5]:

$$
V_{S I F}(\lambda)=\frac{2 \pi}{\lambda} a \sqrt{n_{c o}^{2}(\lambda)-n_{c l}^{2}(\lambda)}
$$

The wavelength dependency of the effective refractive indices is taken into account by introducing the effective index of the fundamental mode, $\mathrm{n}_{\mathrm{co}}(\lambda)$, and of the first cladding mode, $\mathrm{n}_{\mathrm{cl}}(\lambda)$. Instead of an equivalent core radius it is suggested to use the characteristics length scale of the structure namely $(\Lambda)$.

The effective index of the cladding, $\mathrm{n}_{\text {eff, }}$, is assumed a constant value for the microstructure cladding, and can be taken to be that of air, i.e. $\mathrm{n}_{\text {eff }}=1$, for PCFs with cladding consisted of large air holes. In PCFs where the air holes are not large enough for this assumption to be made, $\mathrm{n}_{\mathrm{eff}}$ is approximated as [3]:

$$
n_{\text {eff }}=n_{\text {air }} p_{\text {air }}+n_{\text {silica }} p_{\text {silica }}
$$

where $\mathrm{P}_{\text {air }}$ and $\mathrm{P}_{\text {silica }}$ are the power fraction of air and silica that make up the microstructure cladding respectively, and $\mathrm{n}_{\text {silica }}$ is given by the Sellmeier formula.

Short optical pulses entering a dispersive channel such as an optical fiber are spread out into a much broader temporal distribution and hence dispersion problem will be arising. The total dispersion for the PCF can be determined by applying "(5)", by taking second derivative of the $\left(\mathrm{n}_{\text {eff }}\right)$ given by "(4)" and then applying one of the curve fitting techniques [6].

$$
D_{T}=-\frac{\lambda}{c} \frac{d^{2} n_{e f f}(\lambda)}{d \lambda^{2}}
$$

where $\mathrm{c}$ is the speed of light.
Genetic algorithms (also known evolutionary algorithms) are a class of optimization algorithms that apply principles of natural evolution to optimize a given objective. In genetic optimization of a problem, different solutions to the problem are picked (usually randomly), and a measure of fitness is assigned to each solution [7]. The standard theory of genetic algorithm is based on schema analysis. Schema analysis operates in the representation (genotype) space and can be used provided that the chromosomes chosen are linear strings made up of a fixed number of genes (positions) each of which has a well-defined set of possible alleles (values) and also any combination of alleles is permitted (so that all strings are legal) [8].

On a given generation of the design, a set of operations, analogous to mutation and reproduction in natural selection, are performed on these solutions, which should theoretically be "fitter" than their parents. This process is repeated until the algorithm terminates, typically after a predefined number of generations, or after a particularly "fit" solution is found, or more generally, when a generation of solutions meets some pre-defined convergence criterion [9].

\section{THE PROPOSED METHOD}

In PCF, there is $n_{\text {eff }}$, instead of $n_{\text {cl }}$ which represents the average refractive index in the core layer. The Numerical Aperture (NA) will accept wider range of data comparing to the conventional fiber.

Hence the goal of our work is to determine the propagation characteristics which is the normalized propagation constant (B), or the propagation constant $(\beta)$ with respect to wavelength $(\lambda)$ for the PCF.

To get more accurate results and to become more nearer to the exact solution, a new technique is proposed here by splitting the PCF cable cross section (almost circular) into more than one region (sectors) by taking many rays prolonged from the fiber center toward the cladding with different orientations as shown in Fig. 2. In order to study the dispersion effects, the selected possible profiles of all regions should be taken into account to find out the resultant value of propagation characteristics. Each region bounded between two rays; the first is the horizon ( $\mathrm{x}$-axis), while the second is the ray that makes a certain angle with the horizon; see Fig. 2.

Hence the resultant propagation constant may be determined when all regional profiles are considered. The resultant value may be simply calculated as the average of all individual propagation constants related to each region.

The clad region in the PCF consists of two materials (air and silica), as mentioned earlier, for this reason the $\mathrm{n}_{\mathrm{eff}}$ may represent both materials, and in this work $n_{\text {eff }}$ approximately depends on the PCF cross-section, see Fig. 3.

The most important two parameters that directly affect the total dispersion in such fibers are the number of air-hole layers $(N)$ and the $(\Lambda)$ value that is combined with $(d)$ in $(d$ $/ \Lambda$ ) which is a relative function including the hole diameter (d) and hole-to-hole distance $(\Lambda)$. 


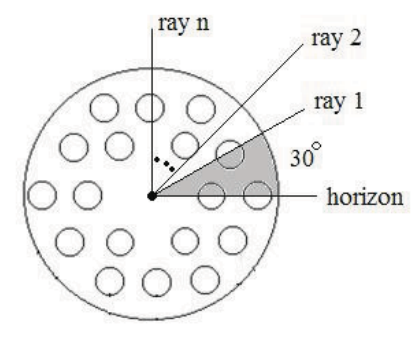

Fig. 2. Split the PCF cross section into many sectors.

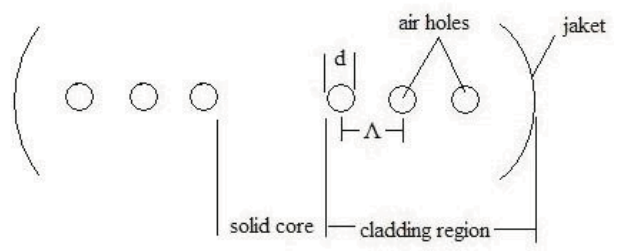

Fig. 3. Simple section of PCF cross-section.

The fiber parameters taken into account during the computation were fixed as follows: $(\Lambda=1.5 \mu, \mathrm{d} / \Lambda=0.75$ and $\mathrm{N}=5)$. The relative difference between $\mathrm{n}_{\text {silica }}$ and $\mathrm{n}_{\text {eff }}$, is shown in Fig. 4.

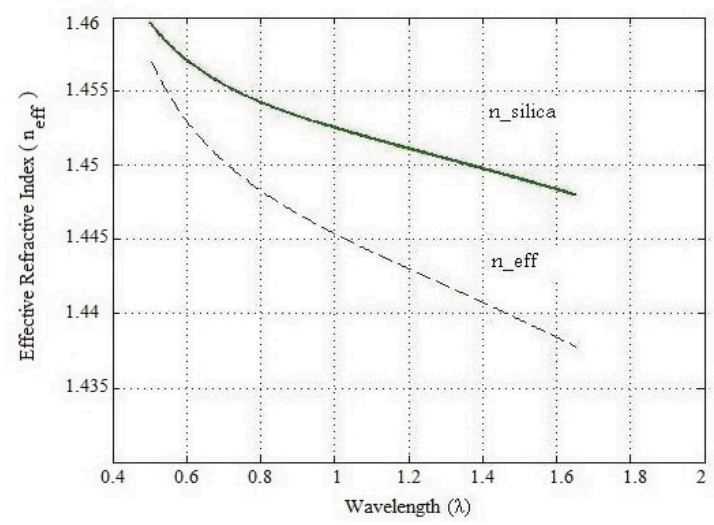

Fig. 4. The relation between $n_{\text {silica }}$ and $n_{\text {eff }}$.

Using the PCF structure of Fig. 3, and after simple mathematical operations, "(4)" may be rewritten as:

$$
n_{\text {eff }}(\lambda)=n_{\text {air }} \frac{(d / \Lambda) N}{0.5 d / \Lambda}+n_{\text {silica }}(\lambda) \frac{(1-d / \Lambda) N+0.5 d / \Lambda}{(0.5 d / \Lambda)+N}
$$

Hence the parameters that directly affect the dispersion and should be taken into consideration in the design are:

1. The profile shape of the PCF (the hexagonal is one of the most popular one).

2. Number of air-hole layers $(\mathrm{N})$.
3. Distance between two adjacent air holes, $(\Lambda)$.

4. Diameter of air hole, $d$.

For given parameters, $\mathrm{n}_{\mathrm{eff}}$ may be calculated versus nsilica at some specific wavelength. The proposed method could be applied to compute the propagation constant for different possible profiles when the following suggested orientation were adopted: $\left(0^{\circ}, 15^{\circ}, 30^{\circ}, 45^{\circ}, 60^{\circ}, 75^{\circ}\right.$ and $\left.90^{\circ}\right)$. Fig. 5 gives the profile of $\left(0^{\circ}\right.$ and $\left.60^{\circ}\right)$ orientations as they are the same as well as the $\left(30^{\circ}\right.$ and $\left.90^{\circ}\right)$ orientations which are identical. On the other hand, Fig. 6 represents the $\left(15^{\circ}, 45^{\circ}\right.$ and $75^{\circ}$ ) orientations.

\section{RESULTS}

To apply the proposed method, computation of PCF fiber with following parameters were adopted: hexagonal structure with $\mathrm{N}=5, \Lambda=1.5 \mu$, and $\mathrm{d} / \Lambda=0.75$, and for different orientation mentioned earlier. To demonstrate the validity of the GA and its usefulness, the effect of various profiles (at different orientations) were investigated. Fig. 7 illustrates the relation between the normalized propagation constant, $\mathrm{B}$, and the wavelength for the suggest PCF for two $\left(0^{\circ}\right.$ and $\left.30^{\circ}\right)$ orientations.

It is apparent from this figure that there is a considerable difference which is increases toward higher wavelengths.

Fig. 7 illustrates the relation between the normalized propagation constant, $\mathrm{B}$, and the wavelength, $\lambda$, for the PCF whose parameters have been shown above but for two orientations, $0^{\circ}$ and $30^{\circ}$, it is apparent from this figure that there is a considerable difference (about 0.12) and it increases toward higher wavelengths. Fig. 8 shows the dispersion characteristics along some specific wavelength range for the same fiber and parameters given above and also for the two mentioned orientations. It is clear that the difference is small in $1.1 \mu \mathrm{m}$ to $1.6 \mu \mathrm{m}$ range while below and above this range it is considerably higher.

Fig. 9 gives clearly the effect the number of layers on the total dispersion, $\mathrm{N}=1,3,5,7$ and 9, while Fig. 10 shows the effect of $\Lambda$ when it equals $1,1.2,1.4,1.6$ and 2 .

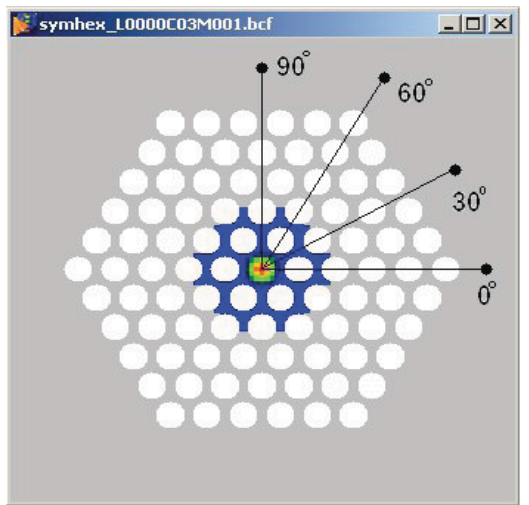



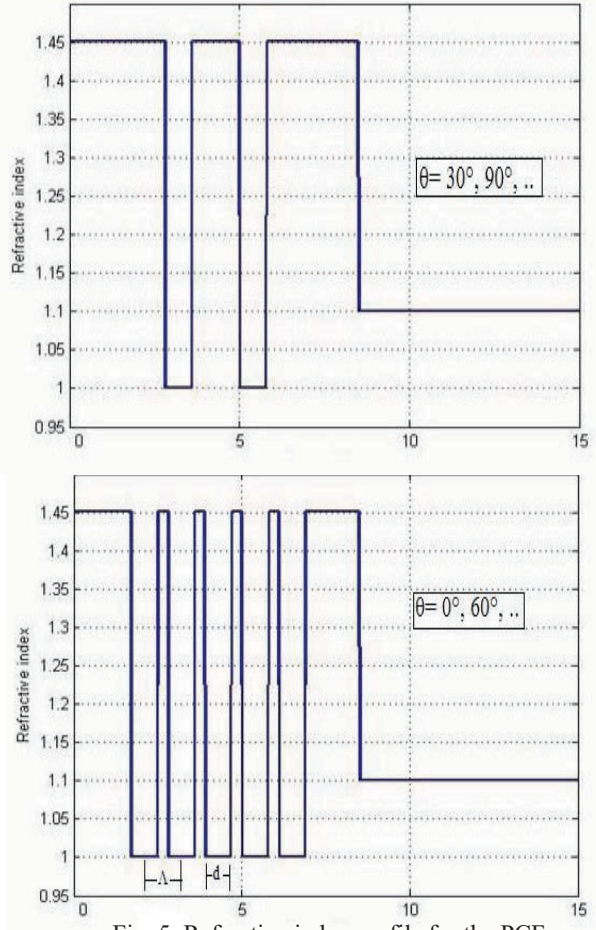

Fig. 5. Refractive index profile for the PCF
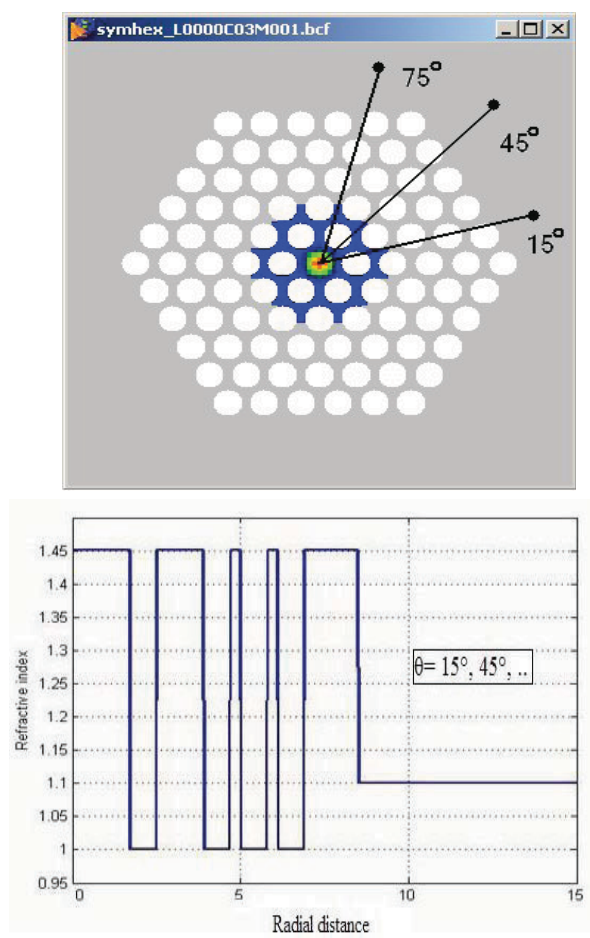

Fig 6. Refractive index profile for the PCF, the angles $15^{\circ}, 45^{\circ}$ and $75^{\circ}$ are considered

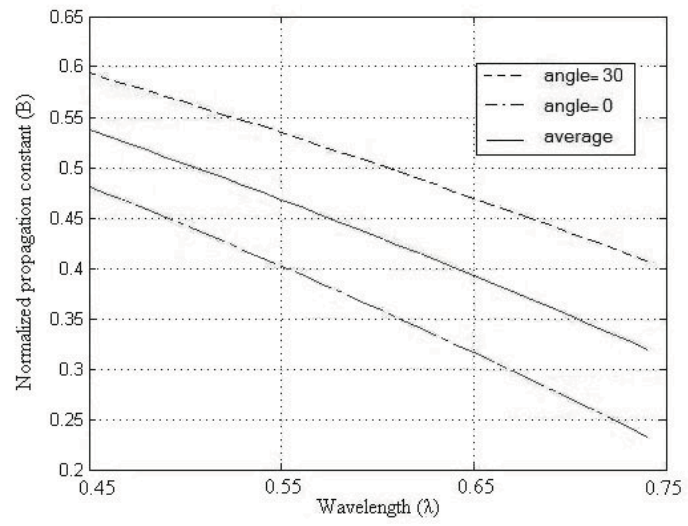

Fig 7. Normalized propagation constant versus wavelengths for the PCF profile with two orientations, ( $\Lambda=1.5 \mu, \mathrm{d} / \Lambda=0.75$ and $\mathrm{N}=5$ ).

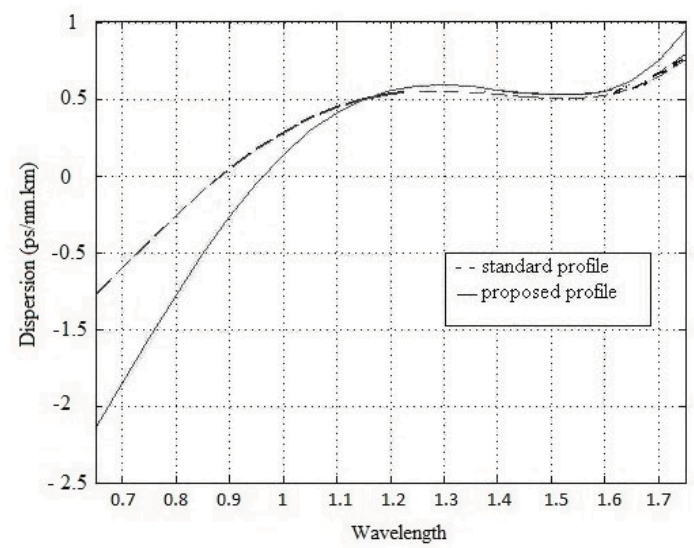

Fig. 8. Dispersion along a wavelength range for the same fiber of Fig. 7.

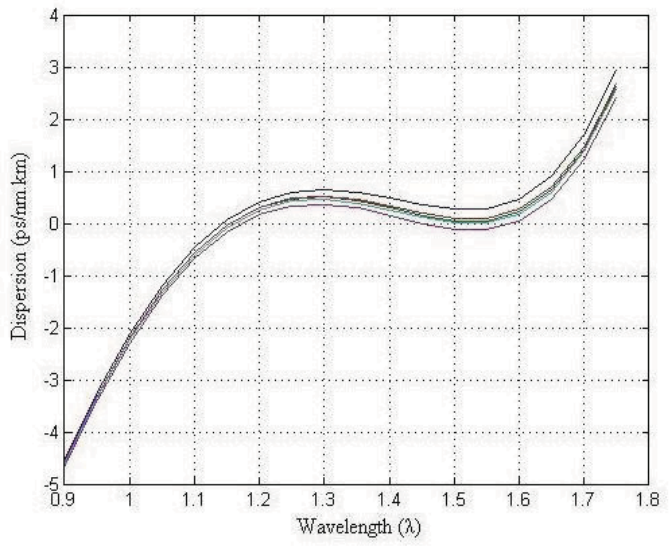

Fig 9. Effect of No. of Air-hole Layers on the Total Dispersion ( $N=1,3,5,7$ and 9) from Top to Bottom. 


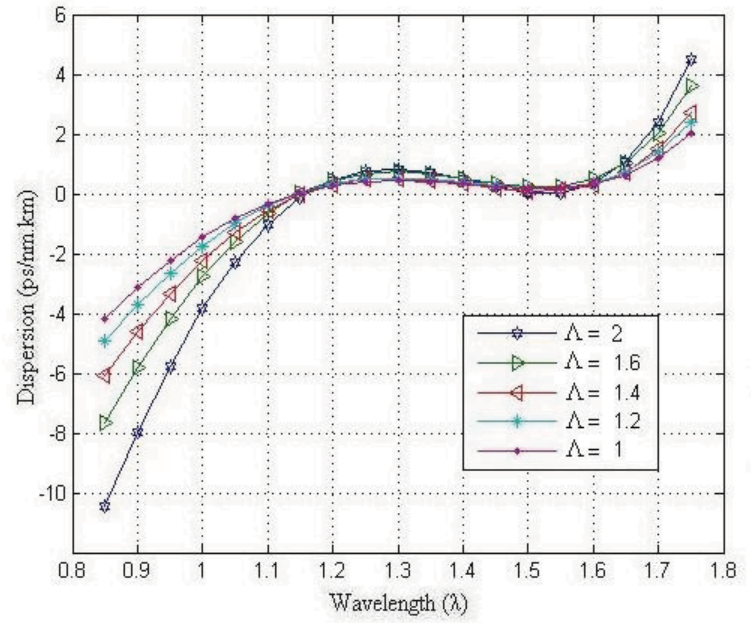

Figure 10. Effect of $(\Lambda)$ on the Total Dispersion, Optimum Case at Smallest Value.

\section{CONCLUSION}

Many researchers have proposed a unique approximation profile to represent each PCF. In this work, a more realistic consideration for PCFs profiles is adopted. Since the materials of such fibers are not homogenous, the special hexagonal structure of PCF with class core and air holes has different shapes when taken the orientation (the angle between horizontal axis and a special ray) into consideration. Hence this difference in profile shape will yield considerable change in the resultant dispersion which should be taken during design process of PCFs.

Using the GAs make the modification very simple and can easily consider any refractive index profile or any electrical field representation.

\section{REFERENCES}

[1] J. Leagsgaard, S. Barkou, K. Hougaard, M. Nielsen, J. Jensen and A. Bjarklev. "Dispersion Properties of Photonic Crystal Fiber - Issues and Opportunities". Mat. Res. Soc. Symp. Proc. VOL. 797, (2004).

[2] X. Feng, T. Monro, P. Petropoulos, V. Fenazzi and D. Hewak. "Solid Microstructured Optical fiber". Optics express Vol. 11, No. 18, (2003).

[3] J. Ssu Yin Chen, "Optical Parametric Amplification in Photonic Crystal fibers". Master of Science thesis, University of Auckland, (2006)

[4] Y. Ni, L. Zhang, L. An, J. Peng and C. Fan. "Dual-Core Photonic Crystal fibers for Dispersion Compensation". IEEE Photonic Technology Letter, VOL. 16, No. 6, (2004).

[5] K. Saitoh, Y. Tsuchida, and M. Koshiba. "Endlessly Single-Mode Holy Fibers: the Influence of Core Design”. Optics Express VOL. 13, No. 26, (2005).

[6] T. Niemi. "Dispersion Measurements of Fiber Optic components and Application of a Novel Tunable Filter for Optical Communication". Master of Science Thesis, Helsinki University of Technology, (2002).

[7] M. Mitchell. "An Introduction to Genetic Algorithms". Massachusetts London, England, (1996).

[8] N. Radcliffe. "The Algebra of Genetic Algorithms". Annals of Maths and Artificial Intelligence, VOL. 10, pp 339-384, (1994).

[9] C. Magnus. "Evolving Waveforms with Genetic Algorithms". Master of Science Thesis, University of California, San Diego, (2003). 